\title{
SÉCULO XXI: NOVA CULTIVAR DE GOIABEIRA DE DUPLA FINALIDADE ${ }^{1}$
}

\author{
FERNANDO MENDES PEREIRA², CELSO ALBANO CARVALHO ${ }^{3}$, JAIR COSTA NACHTIGAL ${ }^{4}$
}

RESUMO - A goiabeira (Psidium guajava L.) é uma espécie que vem se tornando de grande importância em diversas regiões do Brasil, principalmente no Estado de São Paulo, maior produtor nacional. Desde 1985, a UNESP/FCAV, Câmpus de Jaboticabal, vem desenvolvendo um programa de melhoramento genético da goiabeira, com o objetivo de obter plantas com boas características agronômicas e com frutos que possam ser destinados tanto à industrialização quanto ao consumo na forma de fruta fresca. Partindo-se de 219 plantas, oriundas de diversos cruzamentos, e após dez anos de avaliação, chegou-se à cultivar Século XXI, cujas principais características são: planta muito produtiva com ciclo precoce (130 dias da floração à colheita), frutos grandes, com polpa espessa, róseo-avermelhada, ótimo sabor e com poucas e pequenas sementes.

Termos para indexação: Psidium guajava, seleção, melhoramento genético.

\section{CENTURY: NEW CULTIVAR OF GUAVA TREE TO DOUBLE PURPOSE}

ABSTRACT - The guava tree (Psidium guajava, L.) is a species that is coming into being of great importance in several areas of Brazil, mainly in State of São Paulo, its biggest national producer. Since 1985, the UNESP/FCAV, Jaboticabal Campus comes developing a program of breeding of guava tree, with the objective of obtaining plants with good agronomic characteristics and fruits that can be destined for industrialization and consumption as fresh fruit. From 219 plants originating from several crossing, and after ten years of evaluation, it has been obtained the XXI Century cultivar, which main characteristics are: a very productive plant with precocious cycle (130 days from the blossom to the harvest), big fruits with thick pulp, rosy-red, great flavor and with little and small seeds.

Index terms: Psidium guajava, breeding, genetic improvement.

\section{INTRODUÇÃO}

A goiabeira (Psidium guajava, L.) é originária da região tropical do continente americano, com centro de origem, provável, na região compreendida entre o sul do México e o norte da América do Sul. Hoje, esta espécie encontra-se amplamente difundida por todas as regiões tropicais e subtropicais do mundo (Medina, 1988).

A goiaba apresenta lugar de destaque entre as frutas tropicais, principalmente devido ao seu valor nutritivo, com elevados teores de vitamina $\mathrm{C}, \mathrm{A}$ e $\mathrm{B}$, e ao sabor e aroma característicos, que lhe conferem excelente qualidade organoléptica (Pereira \& Martinez Jr., 1986).

Em 1995, o Brasil apresentava uma área de 8.787ha, com uma produção de 255.984 toneladas. Atualmente, a região Sudeste é a maior produtora, com destaque para o Estado de São Paulo, com 4.084ha e uma produção de 151.285 toneladas (Agrianual, 1999).

No Estado de São Paulo, a produção de goiaba para processamento industrial localiza-se, principalmente, nos municípios de Taquaritinga, Ibitinga e Monte Alto. A goiaba para mesa é cultivada principalmente em municípios situados em um raio de 100km da Capital, destacando-se Atibaia, Mogi das Cruzes, Itu e Valinhos (Carvalho, 1996).

A valorização do produto como matéria-prima para a indústria e o aumento de consumo na forma de fruta para mesa têm proporcionado mudanças no sistema de produção e de comercialização. Com isso, torna-se necessário o uso de variedades que atendam às exigências do mercado, tanto para mesa quanto para a indústria (Tavares, 1993).

Um trabalho de melhoramento genético da goiabeira, por meio de seleção de plantas originadas por sementes, pode possibilitar a obtenção de cultivares com características adequadas para o consumo como fruta fresca e para a industrialização (Pereira, 1984).

Com esta finalidade, são selecionadas plantas vigorosas e produtivas, com boa adaptação, produtoras de frutos de boa aparência, com polpa vermelha, e que apresentem grande valor nutritivo, especialmente ricos em açúcares, sais minerais e vitaminas A e C (Pereira, 1995).

O presente trabalho teve por objetivo o desenvolvimento de novas cultivares de goiabeira, com características tais que permitam sua utilização tanto para a industrialização quanto para os mercados de frutas frescas.

\section{MATERIALEMÉTODOS}

O trabalho foi desenvolvido no Pomar Experimental da Faculdade de Ciências Agrárias e Veterinárias, da Universidade Estadual Paulista, Câmpus de Jaboticabal, localizado a $21^{\circ} 15^{\prime}$ de latitude Sul, $48^{\circ} 18^{\prime}$ de longitude Oeste e $595 \mathrm{~m}$ de altitude. O clima da região, segundo a classificação de Köeppen, é do tipo CWa, ou seja, subtropical úmido com chuvas no verão e estiagem no inverno. O solo da área experimental é um Latossolo Vermelho-Escuro de textura argilosa.

Foram estudadas 219 plantas obtidas de quatro cruzamentos realizados por meio de polinização cruzada entre cinco variedades. Os cruzamentos foram os seguintes: 8501 - cultivares Rica x EEF-3; 8502 cultivares Supreme-2 x Paluma; 8503 - cultivares Rica x Patillo 5, e 8504 - cultivares Paluma x Rica.

Com as sementes extraídas de frutos oriundos da polinização cruzada, produzidas nas plantas receptoras do pólen, procedeu-se a formação das mudas. O plantio para o local definitivo foi realizado em 1987, utilizando-se de 25 plantas do cruzamento 8501 , 50 plantas do cruzamento 8502 , 91 plantas do cruzamento 8503 e 53 plantas do cruzamento 8504 .

Durante as fases de implantação e condução do pomar, foram realizados adubações, controle de pragas e doenças, controle das plantas invasoras e podas. Não foram utilizados irrigação e desbaste de frutos em nenhum dos anos de avaliação.

\section{RESULTADOS E DISCUSSÃO}

As avaliações foram iniciadas no ano de 1990, levando-se em consideração características da planta e dos frutos, individualmente em cada cruzamento (Tabelas 1, 2 e 3), conforme metodologia apresentada por Carvalho (1996). Nas safras 1996/97 e 1997/98, foi feita a caracterização tecnológica dos frutos dos melhores genótipos desenvolvidos, conforme metodologia apresentada por Lima (1999) (Tabela 4).

Após dez anos de sucessivas avaliações, chegou-se à seleção

\footnotetext{
(Trabalho 046/2003). Recebido: 01/04/2003. Aceito para publicação: 03/12/2003.

${ }^{2}$ Prof. Titular do Departamento de Produção Vegetal da Faculdade de Ciências Agrárias e Veterinárias, Universidade Estadual Paulista (FCAV/UNESP), Câmpus de Jaboticabal. Via de Acesso Prof. Paulo Donato Castelane, km5, CEP 14870-000, Jaboticabal, São Paulo.

${ }^{3}$ Aluno do Curso de Pós-graduação em Agronomia da Faculdade de Ciências Agrárias e Veterinárias, Universidade Estadual Paulista, Câmpus de Jaboticabal-SP.

${ }^{4}$ Pesquisador da EMBRAPA/CNPUV, Estação Experimental de Jales. Aluno do Curso de Pós-Graduação em Agronomia da Faculdade de Ciências Agrárias e Veterinárias, Universidade Estadual Paulista, Câmpus de Jaboticabal.
} 
TABELA 1 - Características das plantas mais promissoras do Cruzamento Jab 8502 (Supreme-2 x Paluma) durante três safras consecutivas (1990-1993). Jaboticabal, 1996.

\begin{tabular}{cccc}
\hline $\mathrm{N}^{\mathrm{o}}$ da Planta & Época produção & Vigor & Produção \\
\hline 01 (Século XXI) & Precoce & Médio & Boa \\
08 & Mediana & Bom & Boa \\
11 & Precoce & Bom & Boa \\
21 & Tardia & Bom & Boa \\
38 & Mediana & Bom & Boa \\
47 & Mediana & Bom & Boa \\
\hline
\end{tabular}

da planta 8502-01, que recebeu a denominação de Século XXI, e que apresenta as características de planta e de frutos mais adequadas à utilização como fruta fresca, podendo ser amplamente aproveitada para a industrialização.

As plantas da cultivar Século XXI são tão ou mais produtivas que a cultivar Paluma (acima de 30 ton.ha ${ }^{-1}$ ), com ramificações de crescimento predominantemente horizontal e de médio vigor, o que pode permitir a implantação dos pomares utilizando-se de espaçamento menor do que o comumente recomendado para a cultivar Paluma, que é de $7 \mathrm{x}$ $6 \mathrm{~m}$.

A cultivar Século XXI apresenta maturação precoce (Tabela 1), com ciclo de cerca de 130 dias desde a floração até a maturação dos frutos, comparado com a cultivar Paluma, cujo ciclo é de cerca de 158 dias (Pereira, 1995).

Uma das principais características desta cultivar é o grande tamanho de seus frutos (Tabelas 2 e 4), principalmente se considerarmos que, na planta em estudo, não são realizados tratos culturais, como o desbaste de frutos e irrigações, normalmente utilizados em pomares comerciais de goiabas para mesa. O tamanho dos frutos, o ótimo sabor e o bom aspecto são algumas características mais importantes para o mercado de fruta fresca, pois goiabas maiores e saborosas são preferidas pelo consumidor e, por isso, alcançam melhores preços no mercado
(Kavati, 1997).

Os frutos apresentam rendimento de polpa firme de $76 \%$ (Tabela 3), o que está acima de 70\%, mínimo considerado para a seleção de frutos de goiabeira (Lima, 1999), e espessura do pericarpo acima de $160 \mathrm{~mm}$, que confere não apenas boa resistência ao fruto, mas principalmente ótima aparência.

O formato do fruto é ovóide e com pescoço de tamanho reduzido (Tabela 2). Estas características foram alcançadas durante o processo de seleção, a fim de obter um fruto mais resistente aos danos mecânicos, principalmente durante as fases de colheita, classificação, embalagem e comercialização. Segundo Kavati (1997), o formato da fruta pode determinar melhor aspecto visual ao conjunto embalagem/fruta.

A cultivar Século XXI apresenta poucas sementes e com tamanho reduzido (Tabelas 2 e 4). Esta característica aumenta seu valor na indústria, pois possibilita um maior rendimento industrial, bem como qualifica sua apreciação para consumo na forma de fruta fresca, porque as sementes tornam-se menos perceptíveis durante a mastigação.

Durante os anos de avaliação, esta cultivar apresentou mais de $80 \%$ dos frutos com ausência de manchas no pericarpo e sem células pétreas, responsáveis por prejuízos na aparência interna dos mesmos. Tais características são de ordem fisiológica e estão associadas ao desenvolvimento do fruto, portanto sua eliminação é bastante difícil de ser realizada.

Com relação à coloração externa (cor da epiderme), os frutos apresentam-se verde-amarelados, com intenso brilho, contrastando com a coloração rosa, intensa e brilhante, da parte interna, o que lhes confere ótima aparência.

Com relação à composição química (Tabelas 3 e 4), os frutos apresentam teores de sólidos solúveis totais próximos a $10^{\circ} \mathrm{Brix}$, considerados como padrão, e acidez titulável de $0,474 \mathrm{~g}$ de ácido cítrico. $100 \mathrm{~g}_{\text {de }}$ polpa $^{-1}$, o que resulta numa relação SST/AT próximo a 20,0 $(18,6)$, que confere aos frutos sabor bastante adocicado. Segundo Pereira (1995), teores de sólidos solúveis entre 8 e $12^{\circ}$ Brix e acidez

TABELA 2 - Características dos Frutos das mais promissoras plantas do Cruzamento Jab 8502 (Supreme-2 x Paluma) durante três safras consecutivas (1990-1993). Jaboticabal, 1996.

\begin{tabular}{|c|c|c|c|c|c|c|c|c|c|c|c|c|}
\hline $\mathrm{N}^{\mathrm{o}}$ da Planta & Peso (g) & Comp. $(\mathrm{cm})$ & Larg. (cm) & Forma & Firmeza & Sabor & Casca & Cor & Rugos & Semente & $\mathrm{N}^{\mathrm{o}}$ & Tam \\
\hline 01 (Século XXI) & 236 & 9,13 & 7,03 & $\mathrm{OPC}$ & Firme & Bom & & $\mathrm{AM}$ & $\mathrm{R}$ & & $\mathrm{P}$ & $\mathrm{P}$ \\
\hline 08 & 156 & 7,45 & 6,42 & OPC & Firme & Bom & & AM & $\mathrm{L}$ & & $\mathrm{P}$ & $\mathrm{P}$ \\
\hline 11 & 154 & 6,99 & 6,39 & APC & Firme & Bom & & $\mathrm{AM}$ & $\mathrm{L}$ & & $\mathrm{P}$ & $\mathrm{P}$ \\
\hline 21 & 169 & 7,08 & 6,85 & APC & Firme & Bom & & $\mathrm{AM}$ & $\mathrm{L}$ & & $\mathrm{P}$ & $\mathrm{P}$ \\
\hline 38 & 170 & 7,70 & 6,39 & $\mathrm{OPC}$ & Firme & Bom & & $\mathrm{AM}$ & $\mathrm{L}$ & & $\mathrm{P}$ & $\mathrm{P}$ \\
\hline 47 & 200 & 7,65 & 6,88 & APC & Firme & Bom & & AM & $\mathrm{L}$ & & M & $\mathrm{P}$ \\
\hline
\end{tabular}

$\overline{\mathrm{OPC}}$ - ovalado-pescoço curto; APC - arredondada-pescoço curto; AM - amarelada; $\quad \mathrm{R}$ - rugosa; $\mathrm{L}$ - lisa; $\mathrm{P}$ - pequena; $\mathrm{M}$ - média.

TABELA 3 - Características da Polpa das mais promissoras plantas do Cruzamento Jab 8502 (Supreme-2 x Paluma) durante três safras consecutivas (1990-1993). Jaboticabal, 1996.

\begin{tabular}{|c|c|c|c|c|c|c|c|}
\hline $\mathrm{N}^{\mathrm{o}} \mathrm{da}$ & \multicolumn{5}{|c|}{ Polpa Firme } & Polpa Mole & Brix \\
\hline Planta & Peso $(\mathrm{g})$ & $\operatorname{Esp}(\mathrm{mm})$ & Cor & Halo & Manchas & Peso (g) & \\
\hline 01 (Século XXI) & 193 & 162 & $\mathrm{RF}$ & M & $\mathrm{S} / \mathrm{P}$ & 42,75 & 7,23 \\
\hline 08 & 119 & 100 & RF & M & $\mathrm{S} / \mathrm{P}$ & 41,67 & 8,41 \\
\hline 11 & 120 & 129 & V & M & $\mathrm{S} / \mathrm{P}$ & 33,29 & 7,01 \\
\hline 21 & 117 & 132 & B & M & $\mathrm{N}$ & 39,76 & 7,35 \\
\hline 38 & 133 & 139 & RF & M & $\mathrm{S} / \mathrm{P}$ & 36,87 & $\mathbf{8 , 8 9}$ \\
\hline 47 & 161 & 147 & $\mathrm{RF}$ & $\mathrm{F}$ & $\mathrm{S} / \mathrm{P}$ & 39,17 & 8,19 \\
\hline
\end{tabular}

$\mathrm{RF}$ - rosa forte; $\mathrm{V}$ - vermelha; $\mathrm{B}$ - branca; $\mathrm{M}$ - médio; $\mathrm{F}$ - fino; $\mathrm{S} / \mathrm{P}$ - sim/poucas; $\quad \mathrm{N}$ - ausentes.

TABELA 4 - Peso, comprimento e largura dos frutos, espessura da polpa, peso de 100 sementes, sólidos solúveis totais, acidez titulável e vitamina C em frutos das cultivares Rica, Paluma e Jab 8502-01, na safra de 1997-1998. Jaboticabal, 1999.

\begin{tabular}{ccccccccc}
\hline Cultivar & Peso $(\mathrm{g})$ & Compr. $(\mathrm{cm})$ & Largura $(\mathrm{cm})$ & Espessura polpa $(\mathrm{mm})$ & Peso sementes $(\mathrm{g})$ & SST & AcT & Vit. C \\
\hline Rica & 119,4 & 6,4 & 6,1 & 108,0 & 1,6 & 9,4 & 0,53 & 107,0 \\
Paluma & 187,6 & 8,1 & 6,9 & 141,0 & 1,7 & 9,6 & 0,52 & 57,7 \\
8502-01 (Século XXI) & 201,1 & 8,1 & 7,2 & 161,0 & 1,3 & 9,8 & 0,47 & 84,9 \\
\hline
\end{tabular}

SST - sólidos solúveis totais ( ${ }^{\circ}$ Brix); AcT - acidez total titulável (g de ácido cítrico x 100g de polpa); Vitamina C (mg de ácido ascórbico x $100 \mathrm{~g}$ de polpa). 
titulável em torno de $0,8 \mathrm{~g}$ de ácido cítrico. $100 \mathrm{~g}$ de polpa ${ }^{-1}$ são considerados satisfatórios para as diferentes cultivares de goiabeira.

Nos quatro anos de avaliação, o teor de vitamina $C$ encontrado ficou próximo a $100 \mathrm{mg}$ de ácido ascórbico. $100 \mathrm{~g}$ de polpa ${ }^{-1}$, índice superior ao encontrado na cultivar Paluma, utilizada como padrão de seleção para esta característica.

Convém salientar que os teores de SST, AT e vitamina C podem variar de acordo com diversos fatores, principalmente com o grau de maturação utilizado durante as análises. Para fazer a caracterização físicoquímica da cultivar Século XXI, foram utilizados frutos no ponto de colheita comercial, que é caracterizado pela mudança de coloração da epiderme (casca).

\section{CONCLUSÃO}

A cultivar Século XXI, graças às boas características de sua planta e de seus frutos, apresenta amplas possibilidades de cultivo comercial como goiabeira de dupla finalidade.

\section{REFERÊNCIAS BIBLIOGRÁFICAS}

AGRIANUAL 99: anuário da agricultura brasileira. São Paulo: FNP Consultoria \& Comércio, 1999. p.331-335.

CARVALHO, C.A. Seleção de novos cultivares de goiabeira (Psidium guajava L.) através de cruzamentos controlados. 1996. $93 \mathrm{f}$. Dissertação (Mestrado) - Faculdade de Ciências Agrárias e Veterinárias, Universidade Estadual Paulista, Jaboticabal, 1996.

KAVATI, R. Cultivares. In: SIMPÓSIO BRASILEIRO SOBREA CULTURA DA GOIABEIRA, 1., 1997, Jaboticabal. Anais... Jaboticabal: FCAVUNESP/FUNEP/GOIABRAS, 1997. p.1-16.

LIMA, M.A. Conservação pós-colheita de goiaba e caracterização tecnológica dos frutos de diferentes genótipos, produzidos em Jaboticabal, SP. 1999, 101f. Dissertação (Mestrado) - Faculdade de Ciências Agrárias e Veterinárias, Universidade Estadual Paulista, Jaboticabal, 1999.

MEDINA, J.C. Cultura. In: INSTITUTO DE TECNOLOGIAS DE ALIMENTOS. Goiaba. 2. ed. Campinas: ITAL, 1988. p.1-21.

PEREIRA, F.M. A cultura da goiabeira. Jaboticabal: Funep, 1995. 47p.

PEREIRA, F.M. Rica e Paluma: novas cultivares de goiabeira. In: CONGRESSO BRASILEIRO DE FRUTICULTURA, 7., 1984, Florianópolis. Anais... Florianópolis: SBF, 1984. p.524-528.

PEREIRA, F.M.; MARTINEZ JR., M. Goiabas para industrialização. Jaboticabal: Legis Suma, 1986. 142p.

TAVARES, J.C. Efeitos da refrigeração, cera, fungicida e cálcio na conservação pós-colheita da goiaba 'Paluma' (Psidium guajava, L.). 1993. 93f. Tese (Doutorado) - Faculdade de Ciências Agrárias e Veterinárias, Universidade Estadual Paulista, Jaboticabal, 1993. 